\title{
Focal Liver Lesion Tracking in CEUS for Characterisation based on Dynamic Behaviour
}

\author{
Spyridon Bakas ${ }^{1}$, Andreas Hoppe ${ }^{1}$, Katerina Chatzimichail ${ }^{2}$, Vasileios \\ Galariotis $^{2}$, Gordon Hunter ${ }^{1}$, and Dimitrios Makris ${ }^{1}$ \\ 1 Digital Imaging Research Centre, School of Computing and Information Systems, \\ Faculty of Science, Engineering and Computing, Kingston University, Penrhyn Road, \\ Kingston-upon-Thames, Surrey, KT1 2EE, London, United Kingdom \\ \{<s.bakas, g.hunter, d.makris> Qkingston.ac.uk\} \\ 2 Radiology \& Imaging Research Center, University of Athens, Evgenidion Hospital, \\ Papadiamantopoulou Street 20, T.K. 115 28, Athens, Greece. \\ \{katerina@hcsl.com, vgalariotis@hotmail.com\}
}

\begin{abstract}
This paper presents a methodology for tracking a hypo- or hyper-enhanced focal liver lesion (FLL) and a healthy liver region in a video sequence of a Contrast-Enhanced Ultrasound (CEUS) examination. The outcome allows the differentiation between benign and malignant cases, by characterising FLLs of typical behaviour, according to their Time-Intensity curves. The task is challenging mainly due to intensity changes caused by contrast agents. Initially the ultrasound mask is automatically localised and then the FLL and parenchyma regions are tracked, assuming affine transformations on the image plane, employing the point-based registration technique of Lowe's scale-invariant feature transform (SIFT) keypoints detector. Finally, a quantitative evaluation of the tracking process provides a confidence measure for the characterisation decision.
\end{abstract}

\section{Introduction}

Focal liver lesions (FLLs) refer to a particular condition of hepatic disease, which is the fifth largest cause of death in the UK [1]. FLLs are nodules foreign to the liver anatomy that can be either relatively harmless (benignities), or progressively worsening that can potentially result in death (malignancies). There is much interest from clinicians in the potential for the early distinction of a malignant FLL from a benign one as the former, if diagnosed sufficiently early (i.e. in a premature state), can be healed without performing any surgical operation.

Contrast-Enhanced Ultrasound (CEUS) is an attractive imaging modality for the visualisation of FLL candidates as the equipment it requires, when compared with CT or MRI, is relatively inexpensive [2] and portable, allowing its use in any operating room. CEUS has recently gained acceptance for the detection and characterisation of very small FLLs [3]. CEUS requires the intravenous injection of microbubble contrast agents, offering an enhancement to the brightness intensity of blood flow in an image. The modality's effectiveness, in terms of 
Table 1. Signal of FLL VSs during the phases of a CEUS examination. The "+" sign means that the FLL is brighter than the parenchyma and the "-" sign the reverse.

\begin{tabular}{|c|c|c|c|c|}
\hline \multirow{2}{*}{$\begin{array}{l}\text { VASCULAR } \\
\text { SIGNATURE }\end{array}$} & \multicolumn{2}{|c|}{ Hyper-enhanced } & \multicolumn{2}{|c|}{ Hypo-enhanced } \\
\hline & Unipolar. ID:(a) & Bipolar. ID:(b) & Unipolar. ID:(c) & Bipolar. ID:(d) \\
\hline SIGNAI & & & $1 \quad-$ & {$[\quad-/+$} \\
\hline $\begin{array}{l}\text { TYPICAL } \\
\text { BEHAVIOUR } \\
\text { OF }\end{array}$ & $\begin{array}{c}\text { Benign FLL } \\
\text { (e.g.Haemangioma, } \\
\text { Adenoma, Focal } \\
\text { Nodular Hyperplasia) }\end{array}$ & $\begin{array}{c}\text { Malignant FLL } \\
\text { (e.g. Hepatocellular } \\
\text { Carcinoma, } \\
\text { Metastasis) }\end{array}$ & $\begin{array}{l}\text { Benign FLL } \\
\text { (e.g. Nodules, } \\
\text { Cysts) }\end{array}$ & Benign FLL \\
\hline
\end{tabular}

diagnostic accuracy for evaluation of malignant FLLs exceeds 95\%, according to studies by radiologists [4]. However, few radiologists have been trained to apply this modality and interpret its visual cues.

\subsection{CEUS Examination}

The examination comprises three phases, whose duration vary depending on the physiology of the patient's liver and heart. The total duration of an examination lasts a maximum of 10 minutes, after which a diagnosis can be made [5]. The first phase is characterised by the enrichment of the FLL and the healthy liver area (parenchyma) due to the inflow of the contrast medium. During the second phase, the flow of the medium is stabilised, resulting in no relative change in brightness. Finally, the third phase is characterised by the outflow of the medium.

There are two main vascular signatures (VSs) of FLLs: the hyper-enhanced and the hypo-enhanced [Table 1]. VSs describe the way that an FLL dynamically behaves in comparison to the parenchyma. During the first phase, the hyper-enhanced FLLs are enriched prior to the parenchyma, while in the hypoenhanced category the parenchyma is enriched first. FLLs whose VS is hyperenhanced throughout the three phases are called unipolar hyper-enhanced. In contrast, bipolar hyper-enhanced are those where the contrast medium outflows from the FLL prior to the parenchyma during the third phase. If the VS of an FLL is hyper-enhanced, it is mainly in the third phase where its type (benign or malignant) is identified [6], whereas if the VS is hypo-enhanced then the FLL's type is identified as benign from the first phase [7]. The signal of an FLL's VS describes the FLL's dynamic behaviour. Different signals are linked to different medical conditions. Therefore assigning one of the four different signals [Table 1] to an FLL, is of particular medical importance and assists the radiologist make a reliable diagnosis of an FLL of typical behaviour.

\subsection{Challenges of Image Analysis}

Accurate and reliable tracking of an FLL poses a very challenging task due to significant changes in its apparent 2D size, shape, intensity and motion over 


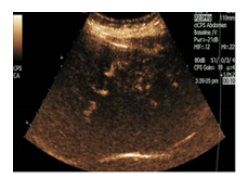

a.(210)

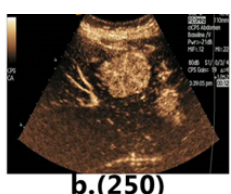

b.(250)

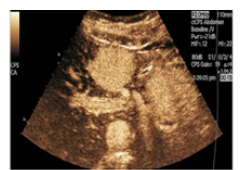

c.(330)

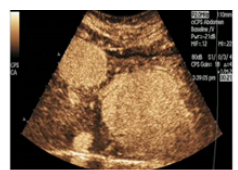

d.(490)

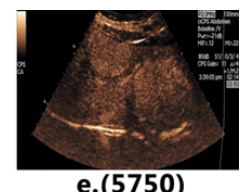

e.(5750)

Fig. 1. Significant changes in the appearance of the liver and the FLL during a CEUS examination. In this particular case, the FLL shape is initialised at frame 250 (b).

the course of the examination (Fig.1). In addition, CEUS imagery is often of low quality, due to noise generated by the propagation of the US waves in soft tissue. This low signal-to-noise ratio, as well as the poor definition of boundaries in the US image makes processing it more complex than images acquired with other imaging modalities (e.g. CT, MRI) [8].

Instability of the clinician's hand holding the transducer and the physiological motion of the patient are additional factors that affect the location of the FLL within an image. Physiological motion refers to the combination of cardiac and breathing motion occurring due to the patient's physiopathology (e.g. variability of heart rate, irregular breathing patterns). This, as well as motion of the inner human organs, affect the motion of the devices and consequently the acquisition of the video, resulting in potential out-of-plane movement. During this movement, a radiologist normally attempts the manual stabilisation of the US target's view within the obtained image plane $(x, y)$ by changing the elevation axis $(z)$ of the US probe, but thus introducing the issue of the FLL's dispersion in depth. Furthermore, the continuous irregular repetitiveness of all these disturbances inevitably degrade the quality of the acquired data.

\subsection{Related Work}

Shiraishi et al. [9] developed a computer-aided diagnostic scheme for distinguishing between only three specific FLL types of hyper-enhanced VS (i.e. Metastasis, Haemangioma and Hepatocellular Carcinoma). Segmentation of the FLLs was obtained by manual annotation of all images processed, by a physician. Then, an Artificial Neural Network was used for the classification of FLLs.

Goertz et al. [6] used the Sonoliver software (Tomtec, Germany) to quantify FLLs. The software allows for semi-automatic motion compensation by combination of manual segmentation of FLLs and parenchyma, background subtraction, and automatic alignment of ROIs, although no specific technical details are given. The outcome of the study [6] implies that the software could only work with data obtained according to particular acquisition standards (e.g. stability of the clinician's hand, no patient's irregular breathing patterns, no FLL dispersion in depth) where motion is minimal.

Bakas et al. [10] developed a histogram-based tracking approach for characterising FLLs of only hyper-enhanced VS. In contrast with [6] and [9], [10] applies a motion tracking approach to the region of interest (ROI) to avoid the need for 
their manual alignment. This approach is mainly based on intensity histograms of the FLL region and also utilises Lowe's scale-invariant feature transform (SIFT) keypoints detector [11]. However, a histogram-based motion tracking approach may suffer because the intensities of the FLL and parenchyma regions become similar towards the end of the first phase and during the second phase. Instead, an automatic motion tracking method based on affine transformations of salient points is proved to be more reliable (see Section 2.2).

\section{Methodology}

The proposed system deals with both VSs of FLLs and attempts to distinguish the type of a FLL (benign or malignant) based on the temporal profile (dynamic behaviour) of its brightness intensity in the image sequence. Robust tracking of two ROIs (FLL and parenchyma) is important, in order to accurately obtain intensity information from the exact areas and therefore provide the ability to characterise with confidence the typical behaviour of FLLs from their dynamic behaviour, while taking into consideration the four main types of VS signals. After obtaining the ground truth (GT) and the decision of the system for these ROIs, a quantitative evaluation of the tracking is carried out, in order to demonstrate that the system can be used as a valid second-opinion tool for a radiologist.

The system employs the point-based registration technique of SIFT [11] to track FLL and parenchyma contours within the conical area covering the ultrasonographic image (US mask), whilst overcoming challenges mentioned in Section 1.2. The derived contours are combined with the statistical analysis method of Generalised Procrustes Analysis (GPA) [12] to model the mean shape of the two ROIs. Size and shape information so obtained are then used to localise these two ROIs in an image from the last phase of the examination and obtain their spatial average intensities at this time $t$. The difference of these average intensities for each frame determine the value of the "signal" of the FLL's VS [Table 1]. Categorising this signal according to pre-defined standard VSs [7] assists the characterisation of the FLL.

\subsection{Definition of Workspace}

In this section, we propose an automatic approach to define the workspace (US mask) (Fig.3.a), rather than to label it manually as in [10]. Therefore, the intensity values of the pixels of the image in a frame $t$, are considered as the visual features $P_{t}$ :

$$
P_{t}=\left[p_{x_{t}, y_{t}}\right]_{W \times H}
$$

where $p_{x_{t}, y_{t}}$ represents the intensity of pixel $p$ with coordinates $(x, y)$ in frame $t$. In addition, $W$ and $H$ depict the width and the height of the image, respectively.

Subsequently, the maximum and minimum intensity values over time are found for each pixel $p_{x_{t}, y_{t}}$ and then the US mask is automatically obtained. The US mask consists of all the pixels satisfying the following criterion:

$$
\max \left(p_{x_{t}, y_{t}}\right)-\min \left(p_{x_{t}, y_{t}}\right)>T_{\text {mask }} .
$$




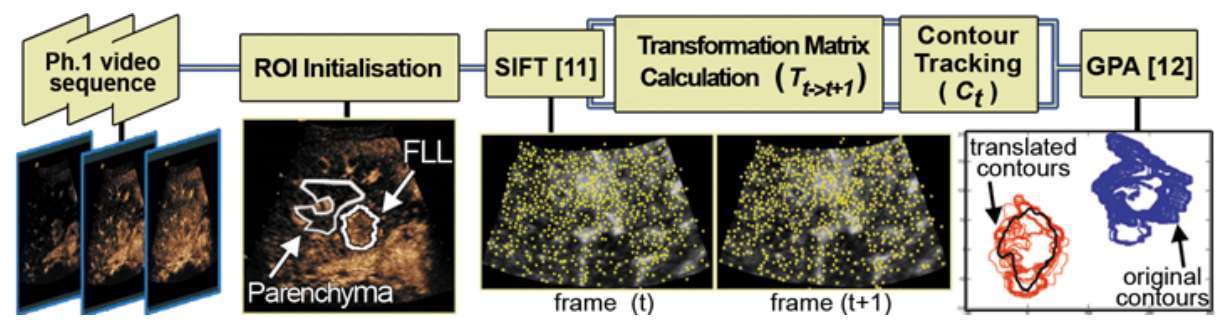

Fig. 2. Phase 1 processing. Where $t \in\left[1, \ldots, N_{f r}\right], N_{f r}=$ numberOfFrames.

where $t \in\left\{1, \ldots, N_{f r}\right\}, N_{f r}$ is the total number of frames and $T_{\text {mask }}$ is a threshold found iteratively after considering that more than one third of the image should be segmented as the foreground area.

The morphological operation of binary erosion is employed to remove protrusions from objects and thin connections between objects. The connected components algorithm is then used to segment these areas. Finally, the largest-sized area within the resulting image provides the final US mask.

\subsection{Motion Tracking under Affine Transformation}

After obtaining the US mask, the video sequence of the first phase is processed to obtain the area and shape descriptors of the ROIs (Fig.2). The FLL and the liver contours (described by a matrix $C_{t}$ ) are initialised by a radiologist at frame $t_{0}$, based on local texture information, as prior medical knowledge is considered advantageous in correctly spotting an FLL (Fig.3.b,c). $C_{t}$ is a $2 \times(U+V)$ matrix, comprising $(U+V) 2 \mathrm{D}$ points, where $U$ and $V$ represent the number of points of the liver and the FLL contour, respectively. The contours are tracked backwards and forwards in time from the frame where they are initialised.

SIFT is used to register salient points $\left(Q_{t}\right.$ and $\left.Q_{t+1}\right)$ within the US mask, in two consecutive frames. $Q_{t}$ is a $2 \times K_{t}$ matrix, comprising $K_{t} 2 \mathrm{D}$ salient points $q_{\kappa, t}$ at time $t$, where $\kappa \in\left[1, K_{t}\right]$. A statistical descriptor vector $\left(D\left(q_{\kappa, t}\right)\right)$ with 128 dimensions is assigned to each point $\left(q_{\kappa, t}\right)$ by SIFT [11], in order to characterise it. The correspondence of the registered points between the two frames is estimated by minimising the Nearest Neighbour Distance Ratio (NNDR) between the descriptors of the two frames. Specifically, for every point $q_{\kappa, t}$, another point $q_{\lambda, t+1}$, where $\lambda \in\left[1, K_{t+1}\right]$, is found that fulfills the following equation:

$$
\left(q_{\kappa, t}, q_{\lambda, t+1}\right)=\underset{\substack{q_{\kappa, t} \in Q_{t} \\ q_{\lambda, t+1} \in Q_{t+1}}}{\operatorname{argmin}}\left[\arccos \left(D^{T}\left(q_{\kappa, t}\right) \bullet D\left(q_{\lambda, t+1}\right)\right)\right]
$$

Similarly, we find $q_{\lambda^{\prime}, t+1}$, where $\lambda^{\prime} \in\left[1, K_{t+1}\right]$, such that:

$$
\left(q_{\kappa, t}, q_{\lambda^{\prime}, t+1}\right)=\underset{\substack{q_{\kappa, t} \in Q_{t} \\ q_{\lambda^{\prime}, t+1} \in Q_{t+1}, \lambda^{\prime} \neq \lambda}}{\operatorname{argmin}}\left[\arccos \left(D^{T}\left(q_{\kappa, t}\right) \bullet D\left(q_{\lambda^{\prime}, t+1}\right)\right)\right]
$$




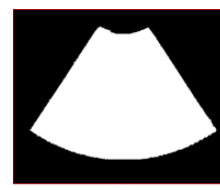

a.

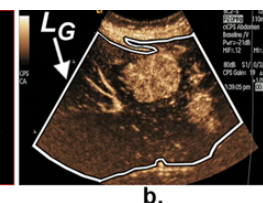

b.

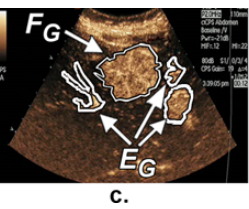

c.

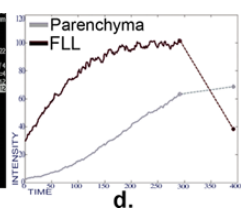

d.

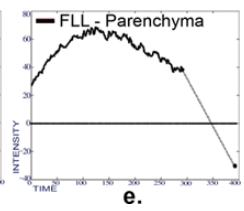

Fig. 3. a.Automatically obtained US mask. b.Liver's GT $\left(L_{G_{t}}\right)$. c.FLL's GT $\left(F_{G_{t}}\right)$ and non-liver areas' GT $\left(E_{G_{t}}\right)$, d.Time-Intensity curves (TIC), e.VS Signal

We accept that there is a correspondence between points $q_{\kappa, t}$ and $q_{\lambda, t+1}$ in the two frames $t$ and $t+1$, if:

$$
\frac{\arccos \left(D^{T}\left(q_{\kappa, t}\right) \bullet D\left(q_{\lambda, t+1}\right)\right)}{\arccos \left(D^{T}\left(q_{\kappa, t}\right) \bullet D\left(q_{\lambda^{\prime}, t+1}\right)\right)}<Z
$$

where $Z \in[0,1]$ is a threshold value. If a point $q_{\lambda, t+1}$ is the best match for more than one point $q_{\kappa, t}$, then these correspondences of $q_{\lambda, t+1}$ are rejected. Consequently, we only keep reliable correspondences.

The deformations on the image plane between these correspondences are described by affine transformations (e.g. translation, rotation, shear). Therefore, they are approximated by a transformation matrix $T_{t \rightarrow t+1}$ that is computed by the following equation:

$$
A_{t+1}=T_{t \rightarrow t+1} A_{t}=>T_{t \rightarrow t+1}=A_{t+1} A_{t}^{-1}
$$

where $A_{t}$ and $A_{t+1}$ are $2 \times R_{t, t+1}$ matrices, comprising $R_{t, t+1} 2 \mathrm{D}$ salient points at time $t$ and their correspondences at time $t+1$, respectively (Similarly with $t$ and $t-1$ respectively, if tracking backwards). As matrix $A_{t}$ is not square, $A_{t}^{-1}$ is its Moore-Penrose inverse [13], computed using the singular value decomposition.

$T_{t \rightarrow t+1}$ is then applied to $C_{t}$, in order to obtain the corresponding contours in the subsequent frame, estimated as $C_{t+1}=T_{t \rightarrow t+1} C_{t}$. Thus, the contours are tracked in subsequent frames, while their shape and size are also updated based on the affine transformation of subsequent frames, instead of applying the global translation of corresponding SIFT points to the centre of each contour as done in $[10]$.

After all ROIs' contours are obtained in all frames, they are sampled to a set of marked points, in order to model the shape of that ROI. The overall mean shape of the sampled contours is computed by GPA [12] by optimally superimposing the set of all the contours on a single reference orientation. As shown in Fig.2 the FLL's contours originally tracked through the sequence are aligned and then their mean shape is obtained (black-contour). Such a shape is expected to be more accurate than the shape defined manually by the radiologist based on local texture information, as the former takes into account variation across all the frames. 

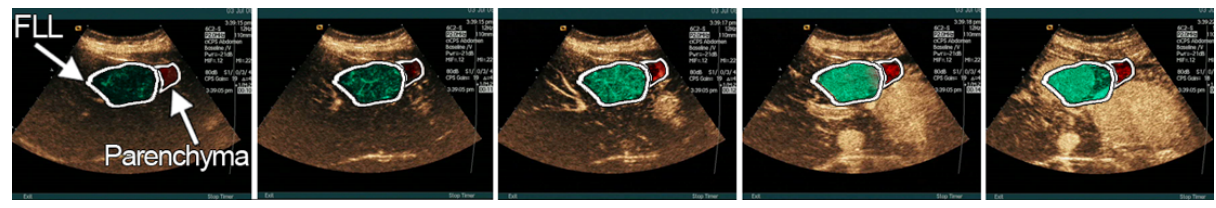

Fig. 4. Motion tracking of the FLL and parenchyma regions with the proposed method.

Finally, the value of the signal of the FLL's VS (Fig.3.e) is determined by the following equation:

$$
\text { Signal }_{V S}=\frac{\sum_{i=1}^{n_{t}} p_{x_{t, i}, y_{t, i}}}{n_{t}}-\frac{\sum_{j=1}^{l_{t}} p_{x_{t, j}, y_{t, j}}}{l_{t}}
$$

where $n_{t}$ and $l_{t}$ are the number of the pixels within the FLL and parenchyma regions respectively, at frame $t$. The characterisation of the FLL according to the four predefined VSs [7] is then based on this signal throughout the duration of the examination.

\section{Results \& Validation}

\subsection{Data Acquisition}

The proposed tracking system is applied and evaluated on real clinical data of 14 case studies of patients with similar physical condition. The data acquisition is done by using a Siemens ACUSON Sequoia C512 system with low-frequency 6C2 convex Transducer $(2-6 \mathrm{MHz})$ capturing $25 \mathrm{fps}$ and following the workflow described in Section 1. Each case includes at least one video sequence from the 1st phase and a static image of the 3rd phase with resolution 768x576 pixels and no compression applied. The contrast medium used was the 2 nd-generation contrast medium of sulphur hexafluoride microbubbles (SonoVue, Bracco Diagnostics) that allows for excellent depiction of the FLL vascularity and perfusion [3].

\subsection{Ground Truth}

To evaluate the accuracy of the tracking method and the level of confidence of the decision on the signal of the FLL's VS, the exact positions that the FLL and the liver areas occupy on each frame were manually annotated, providing their GT as $F_{G_{t}}$ and $L_{G_{t}}$ respectively, for every frame $t$ (Fig.3.b,c).

The initialisation of their shapes is performed by the radiologist, whereas their displacements in subsequent frames is done by another operator. The same shape is used for initialising the FLL at frame $t_{0}\left(C_{t_{0}}\right)$ and creating $F_{G_{t_{0}}}$ in the proposed system. Furthermore, the GT of areas that do not belong to, but appear within the liver have been manually labelled on each frame $t\left(E_{G_{t}}\right)$, in order to take these artefacts (i.e. veins and arteries) into account when evaluating the tracking of the parenchyma (Fig.3.c). 


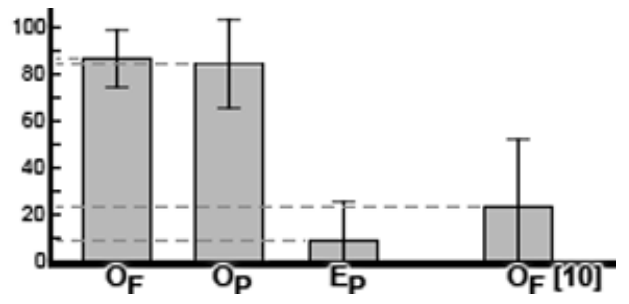

Fig. 5. $O_{F}$ and $O_{P}$ depict the overlap of the FLL and the parenchyma, respectively, as explained in Section 3.3. $E_{P}$ depicts the noise included within $O_{P}$ in our method.

\subsection{Evaluation Metrics}

The accuracy of the proposed method is quantitatively evaluated by comparing its decisions with the GT throughout the sequence. The validation of the system is based on considering five regions: the GTs $\left(F_{G_{t}}, P_{G_{t}}, E_{G_{t}}\right)$, the FLL decision $\left(F_{d_{t}}\right)$ and the parenchyma decision $\left(P_{d_{t}}\right)$. Note that $P_{G_{t}}=F_{G_{t}}^{C} \cap L_{G_{t}}$, where $F_{G_{t}}, P_{G_{t}} \subset L_{G_{t}}$, and $E_{G_{t}} \subset P_{G_{t}}$.

The spatial overlap metric for the FLL regions $\left(O_{F}\right)$ is computed by the equation $O_{F}=\frac{1}{N_{f r}} \sum_{t=1}^{N_{f r}} O_{F_{t}}$, where $O_{F_{t}}=\frac{\left|F_{G_{t}} \cap F_{d_{t}}\right|}{\left|F_{G_{t}} \cup F_{d_{t}}\right|}$. This $\left(O_{F_{t}}\right)$ obtains information only from pixels $p_{x_{t}, y_{t}} \in F_{G_{t}} \cap F_{d_{t}}$ and penalises pixels misclassified as either FLL or non-FLL $\left(p_{x_{t}, y_{t}} \in F_{G_{t}} \triangle F_{d_{t}}\right)$.

The spatial overlap metric of the parenchyma regions $\left(O_{P}\right)$ is computed as $O_{P}=\frac{1}{N_{f r}} \sum_{t=1}^{N_{f r}} O_{P_{t}}$, where $O_{P_{t}}=\frac{\left|P_{G_{t}} \cap P_{d_{t}}\right|}{\left|P_{d_{t}}\right|}$. This $\left(O_{P_{t}}\right)$ obtains information from pixels $p_{x_{t}, y_{t}} \in P_{G_{t}} \cap P_{d_{t}}$, but its definition differs from that for $O_{F_{t}}$, as pixels $p_{x_{t}, y_{t}} \in P_{d_{t}}^{C} \cap P_{G_{t}}$, whenever $P_{d_{t}} \subset P_{G_{t}}$, should not be penalised. This allows the operator to initialise a smaller region for tracking $\left(P_{d_{t}}\right)$, within $P_{G_{t}}$, as correct information will still be provided taking into account the spatial uniformity of the enrichment distribution of $P_{G_{t}}$. Last but not least, there is a need to evaluate the percentage of pixels that are non-liver areas but incorrectly classified as parenchyma (i.e. $E_{P_{t}}$ ), which are computed as follows: $E_{P}=\frac{1}{N_{f r}} \sum_{t=1}^{N_{f r}} E_{P_{t}}$, where $E_{P_{t}}=\frac{\left|E_{G_{t}} \cap P_{d_{t}}\right|}{\left|P_{d_{t}}\right|}$

\subsection{Results}

In the cases provided, the third phase is depicted only in static images. Therefore, the FLL ROI is localised in the third phase by employing the exhaustive search method of sliding window and maximising a spatial overlap criterion as in [10]. More specifically, the ROI is localised by maximising the intersection between the automatically segmented areas of the static image and the FLL's mean shape as estimated by GPA in the first phase, allowing translation and rotation of the latter through a sliding window. 
Table 2. FLL characterisation. VS ID refers to IDs given in Table 1 (e.g.(a),(b),(c),(d)).

\begin{tabular}{|c|c|c|c|c|c|c|c|c|c|c|c|c|c|c|}
\hline Case studies & 1 & 2 & 3 & 4 & 5 & 6 & 7 & 8 & 9 & 10 & 11 & 12 & 13 & 14 \\
\hline VS ID (GT) & (b) & (b) & (b) & (b) & (a) & (a) & (a) & (b) & (b) & (b) & (b) & (b) & $(c)$ & (c) \\
\hline VS ID (Proposed System) & (b) & (b) & (b) & (b) & (a) & (a) & (a) & (b) & (b) & (b) & (b) & (b) & (b) & (c) \\
\hline VS ID of $[10]$ & (b) & (b) & (b) & (b) & (a) & (a) & (a) & (b) & (b) & - & - & - & - & - \\
\hline
\end{tabular}

An example of the application of the proposed method is shown in Fig.4, demonstrating that the ROIs are tracked even when the video's brightness and contrast are extremely low (e.g. beginning of sequence). The frames show the two ROIs under dramatic appearance changes over time.

The overall average spatial overlaps across our clinical data are above $88 \%$ and $86 \%$ for $O_{F}$ and $O_{P}$ respectively, while for $E_{P}$ is just above $9 \%$ (Fig.5). The mean of $O_{F}$ and $O_{P}$ exceeding $87 \%$ shows the success of the proposed tracking method, giving confidence that the obtained signal of the VS is truly representative of the FLL, hence providing reliable characterisation of FLLs of typical behaviour. As shown in the first two rows of Table 2 this proved to be valid in 13 out of 14 cases. VS of FLL of case 13 is mischaracterised, as incorrect intensity information is obtained due to much increased dispersion in depth.

The method in [10] is evaluated using the same dataset as ours. Note that [10] is unable to track the parenchyma. The results depict $22.4 \%$ overlap $\left(O_{F}[10]\right.$ - Fig.5). This low level of overlap, is caused by the histogram-based approach struggling to deal with the intensity variations and therefore fails to properly update the shape of the FLL over time. On the other side, the hereby proposed method considers salient points' deformations of affine transformations for all the regions' updates, which seems more reliable. The final decision of each method is shown in Table 2. Comparison of the results between the two methods depict that [10] is unable to characterise FLLs of hypo-enhanced VS (cases 13,14) as it was not designed for this VS. Also [10] fails if video has low brightness and contrast (cases 10,11,12). In general, tracking in [10] suffers when the relative differences of brightness and contrast between FLL and parenchyma become small (e.g. towards the end of phase 1 and during phase 2). It should be noted that [10] does not characterise an FLL based on its dynamic behaviour, but by observations on the sign change of the signal of hyper-enhanced FLLs, between phase 1 and 3 .

\section{Conclusion}

The proposed system provides accurate tracking and quantitative evaluation of the FLL and the parenchyma areas, whilst characterising the type of an FLL based on the signal of its VS (dynamic behaviour). The tracking of the FLL and the parenchyma is automated by taking into account deformations of the regions as affine transformations on the image plane and only requires the initialisation of the ROIs in one frame as an input by the operator. In our experiments, the 
ROIs of 14 clinical cases were tracked with average spatial overlap $87.2 \%$ and 13 lesions were correctly characterised by the proposed system.

\section{References}

1. BritishLiverTrust: Website. (http://www.britishlivertrust.org.uk/home/lookingafter-your-liver.aspx [Last accessed 09 December 2011])

2. Sirli, R., Sporea, I., Martie, A., Popescu, A., Danila, M.: Contrast enhanced ultrasound in focal liver lesions-a cost efficiency study. Medical Ultrasonography $\mathbf{1 2}$ (2010) 280-285

3. Wilson, S.R., Burns, P.N.: Microbubble-enhanced us in body imaging: What role? Radiology 257 (Oct. 2010) 24-39

4. Strobel, D., Seitz, K., Blank, W., Schuler, A., Dietrich, C.F., vonHerbay, A., Friedrich-Rust, M., Bernatik, T.: Tumor-specific vascularization pattern of liver metastasis, hepatocellular carcinoma, hemangioma and focal nodular hyperplasia in the differential diagnosis of 1349 liver lesions in contrast-enhanced ultrasound (ceus). Ultrachall in Med 30 (2009) 376-382

5. Albrecht, T., Blomley, M., Bolondi, L., Claudon, M., Correas, J.M., Cosgrove, D., Greiner, L., Jager, K., de Jong, N., Leen, E., Lencioni, R., Lindsell, D., Martegani, A., Solbiati, L., Thorelius, L., Tranquart, F., Weskott, H.P., Whittingham, T.: Guidelines for the use of contrast agents in ultrasound - january 2004. Ultrachall in Med 25 (2004) 249-256

6. Goertz, R.S., Bernatik, T., Strobel, D., Hahn, E.G., Haendl, T.: Software-based quantification of contrast-enhanced ultrasound in focal liver lesions - a feasibility study. European Journal of Radiology 75 (2010) 22-26

7. Rognin, N.G., Mercier, L., Frinking, P., Arditi, M., Perrenoud, G., Anaye, A., Meuwly, J.Y.: Parametric imaging of dynamic vascular patterns of focal liver lesions in contrast-enhanced ultrasound. IEEE International Ultrasonics Symposium (IUS) (2009) 1282-1285

8. Noble, J.A.: Ultrasound image segmentation and tissue characterisation. Proceedings of the Institution of Mechanical Engineers, Part H: Journal of Engineering in Medicine 224 (2010) 307-316

9. Shiraishi, J., Sugimoto, K., Moriyasu, F., Kamiyama, N., Doi, K.: Computer-aided diagnosis for the classification of focal liver lesions by use of contrast-enhanced ultrasonography. Medical Physics 35 (2008) 1734-1746

10. Bakas, S., Chatzimichail, K., Autret, A., Hoppe, A., Galariotis, V., Makris, D.: Localisation and charasterisation of focal liver lesions using contrast-enhanced ultrasonoghaphic visual cues. In Proceedings of Medical Image Understanding and Analysis (2011)

11. Lowe, D.G.: Distinctive image features from scale-invariant keypoints. International Journal of Computer Vision 60 (2004) 91-110

12. Gower, J.: Generalized procrustes analysis. Psychometrika 40 (1975) 33-51

13. Penrose, R.: A generalized inverse for matrices. Mathematical Proceedings of the Cambridge Philosophical Society 51 (1955) 406-413 